\title{
APLICAÇÃO DE AEROGÉIS PARA REMOÇÃO DE PITCH NA INDÚSTRIA DE CELULOSE E PAPEL
}

\author{
F. S. SILVA ${ }^{1}$, M. O. RODRIGUES ${ }^{2}$, R. C. de OLIVEIRA ${ }^{3}$ \\ ${ }^{1}$ Universidade Federal de Viçosa, Departamento de Química \\ ${ }^{2}$ Universidade Federal de Viçosa, Departamento de Química \\ ${ }^{3}$ Universidade Federal de Viçosa, Departamento de Engenharia Florestal \\ E-mail: fernanda.s918@gmail.com
}

\begin{abstract}
RESUMO: $O$ licor negro formado na polpação da indústria de celulose e papel contém pitchs, materiais resinosos em estado coloidal, derivados da madeira, que se acumulam nos maquinários da indústria, danificando-os ou o produto final. O objetivo do trabalho foi estudar o uso de aerogéis à base de celulose na remoção dos pitchs, função exercida pelo talco na maioria das indústrias. Os aerogéis são sólidos porosos, produzidos a partir de liofilização de uma suspensão coloidal de celulose nanofibrilada e xilanas, em diferentes proporções, e resina PAE - poliamina-epiclorohidrina. A contagem dos pitchs foi feita com uma câmara de Neubauer. Todos os aerogéis apresentaram poder de adsorção, de modo que quanto maior o teor de CNF-Celulose Nanofibrilada, melhor o adsorvente. De modo geral, os aerogéis 100:0 (CNF:Xilana) são melhores adsorventes que os aerogéis 30:70. Não foi observada uma proporção entre massa e capacidade de adsorção, sugerindo a existência de quantidades especificas para cada aerogel.
\end{abstract}

PALAVRAS-CHAVE: Pitch; Aerogel; Celulose; Hemicelulose; Adsorção.

\section{INTRODUÇÃO}

Para a produção de celulose pela indústria há necessidade da conversão da madeira em um material fibroso, através de um processo de polpação. O método mais usado é o processo Kraft ou Sulfato, no qual são utilizados dois reagentes químicos para atuar na transformação da madeira: hidróxido de sódio e sulfeto de sódio. Nesse processo, a madeira reage com o licor branco (reagentes químicos), produzindo o licor negro. O licor negro consiste de uma solução complexa formada por cerca de $60 \%$ de matéria orgânica, como ácidos carboxílicos, extrativos e fragmentos de lignina e cerca de $40 \%$ de matéria inorgânica em base seca, como reagentes não convertidos da polpação e produtos químicos residuais (FORTUNATTO, 2014).

Devido à presença de compostos, tais como resinas, nos extrativos e também de aditivos usados para processar a polpa, depósitos chamados pitchs se formam, segundo Barbosa et al., (2006). Os compostos lipofílicos são os mais problemáticos e incluem ácidos graxos livres, ceras, ácidos resinosos, esteróis, ésteres, cetonas, glicerídeos e outros 
compostos oxidados (GUTIERREZ et al., 2001). Essas substâncias são liberadas durante o cozimento da madeira e algumas delas, como as ceras, esteróis e alguns ésteres não formam sabões solúveis, formando partículas coloidais que podem tanto se depositar na polpa celulósica, quanto nos equipamentos ou então permanecer suspensos em água de processo (DEL RIO et al., 1998 e DEL RIO et al., 2000).

O pitch é o acúmulo destes compostos, ligado a materiais inorgânicos e a fibras em proporções variadas, que se incrusta nos equipamentos podendo formar manchas nas folhas de celulose produzidas e nos papéis formados no processo. Fatores, como estocagem de madeira, condições de cozimento, lavagem de polpa e qualidade da água do processo influenciam na maior ou menor frequência de formação de pitch (SANSIGOLO et al., 2010).

As gotículas de pitchs têm movimento "browniano", movimento constante e desordenado, e são perfeitamente esféricas, diferentemente de outras partículas coloidais da polpa. Outras partículas não perfeitamente esféricas apresentam movimentação com turbulência, evidenciando a sua não perfeita esfericidade, diferenciando-se do "pitch" (CAMARA, 2003).

O talco apresenta propriedades de adsorver materiais oleosos em presença de água. Essa propriedade é extremamente importante para capturar pitchs nas polpas. A diminuição de pitch pelo uso de talco consiste em adsorver os constituintes resinosos, formadores de depósitos e removê-los do processo, de forma que não haja prejuízo de qualidade do produto final (JORDÃO, 1991).

O talco é, porém, um mineral retirado da natureza e, portanto, não renovável. Aerogéis gerados com material celulósico são uma proposta alternativa de substituição ao talco no processo de produção de celulose e papel. O aerogel é um dos materiais de baixa densidade obtido a partir da celulose, polímero orgânico mais abundante do planeta, que conta com uma produção estimada em mais de 7,5 x $10^{10}$ toneladas por ano. (HABIBI et al., 2010).

Os aerogéis são materiais porosos de nanoestrutura feitos a partir da liofilização de soluções de compostos celulósicos, onde há substituição do líquido pelo gás. Esses materiais têm outras aplicações potenciais, nos campos da medicina, cosméticos e farmacêuticos (AULIN, 2010). Neste trabalho foram utilizados aerogéis produzidos segundo a metodologia descrita na tese de doutorado em Silva (2015), a partir de polpa celulósica, constituída de celulose nanofibrilada (CNF), resina poliamina-epiclorohidrina (PAE) e em alguns casos reforçada com hemicelulose.

A celulose nanofibrilada ou nanofibrilas de celulose é obtida a partir da desintegração mecânica de fibras celulósicas. Ela apresenta regiões amorfas e cristalinas associadas à grupos hidroxílicos, que favorecem a formação de várias ligações de hidrogênio (PÄÄKKÖ et al., 2007).

A resina PAE favorece a constituição dos aerogéis devido à sua natureza polimérica, através de ligações cruzadas que reforçam e mantêm a estrutura deles. Como apresenta cargas positivas, a sua utilização favorece a adsorção por interação iônica com os pitch coloidais, normalmente de carga negativa, assim como com outros resíduos que formam o pitch depositável. 
As indústrias de celulose e papel apresentam, com frequência, problemas relacionados ao acúmulo de pitchs coloidais, quer seja nas polpas e papéis formados, quer seja no maquinário utilizado. A revista "O Papel", da Associação Brasileira Técnica de Celulose e Papel - ABTCP - publicou em maio 2016 que a receita de exportações de celulose, painéis de madeira e papel somou US\$ 1,4 bilhão, apresentando um crescimento de $19,7 \%$ em comparação com o mesmo período de 2015. Justifica-se, portanto, a necessidade de estudos sobre controle e remoção dessas micelas nessa indústria, que se mostra promissora no mercado brasileiro.

O objetivo desse trabalho foi de avaliar a aplicação de aerogéis para a remoção de pitch, em substituição ao talco.

\section{MATERIAIS E MÉTODOS}

Os materiais utilizados foram: licor negro industrial diluído $(30 \% \mathrm{v} / \mathrm{v})$ fornecido por uma indústria de celulose Kraft de eucalipto, cedido pelo Laboratório de Celulose e Papel da Universidade Federal de Viçosa (LCP - UFV); talco em pó referência na indústria celulósicopapeleira e aerogéis de polpa branqueada de celulose, cedidos pelo LCP - UFV, e vidrarias em geral.

Os aerogéis utilizados no presente experimento, mostrados na Figura 1, foram produzidos a partir de polpa celulósica e caracterizados segundo a metodologia descrita na tese de doutorado em Silva (2015). Tais aerogéis são constituídos de celulose nanofibrilada (CNF), reforçados com hemiceluloses ricas em xilanas, em diferentes proporções. Além disso, possuem resina poliamina-epiclorohidrina (PAE) na sua constituição, necessária à formação reticular, para controle de resistência à penetração de líquidos e manutenção da forma dos aerogéis em meio aquoso. Os aerogéis produzidos por Silva (2015) a partir de suspensões de CNF, advém de misturas de razões CNF:Hemiceluloses variando de 100:0 a 0:100. Neste trabalho, utilizaram-se os aerogéis de composição 100:0, 70:30 e 30:70.

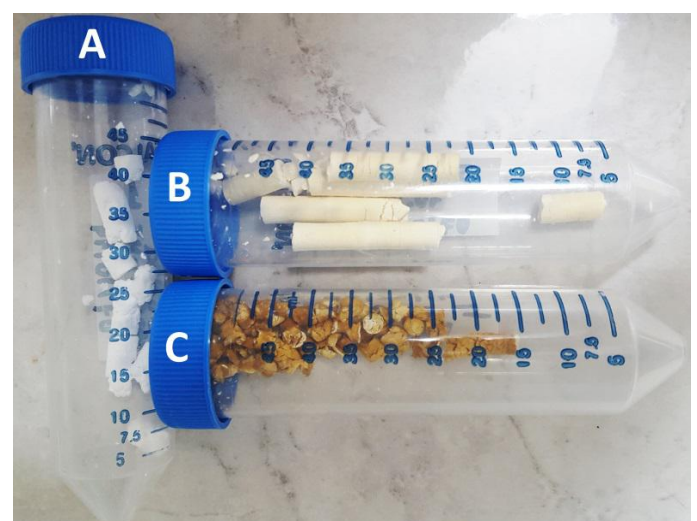

Figura 1 - Aerogéis (CNF:Xilana): A (100:0), B (70:30), C (30:70).

A resina PAE - epiclorohidrina, agente de reticulação, foi adicionada às misturas sob agitação mecânica. Os géis formados por variadas composições foram adicionados a moldes. As amostras foram submersas em nitrogênio líquido durante 5 minutos. Finalmente, as amostras congeladas foram secas num liofilizador, a uma temperatura de sublimação de $-52^{\circ}$ C e uma pressão de 0,160 mbar, durante 44 horas. A reticulação de aerogéis foi conseguida 
por cura das amostras liofilizadas numa estufa de vácuo a $120^{\circ} \mathrm{C}$ durante $3 \mathrm{~h}$.

As áreas superficiais específicas dos aerogéis foram determinadas por medidas de adsorção/dessorção com Nitrogênio. Ambas as isotermas foram medidas e a área superficial foi determinada a partir dos resultados, utilizando o método de Brunauer-Emmet-Teller (BET). Antes das medições, as amostras foram secas a uma temperatura de $105^{\circ} \mathrm{C}$, durante 4 horas (SILVA, 2015).

O licor negro foi utilizado como a fonte de pitch. Para determinar o potencial de adsorção das amostras na remoção das micelas de pitch, foram feitos procedimentos com base na metodologia PAPTAC (2011). Foram utilizados talco e 3 tipos de aerogéis nas seguintes proporções CNF:Xilana: 100:0, 70:30 e 30:70.

Para fins de cálculos e análises, todos os experimentos foram feitos à temperatura ambiente, $25^{\circ} \mathrm{C}$, e pH do licor negro mantido constante e igual a 13.

Para a contagem de micelas de pitch coloidal, seguindo a metodologia PAPTAC (2011), foram usadas amostras de licor contendo talco e aerogéis com as seguintes concentrações:

- $2 \mathrm{~kg}$ de talco/tonelada do licor negro;

- $2 \mathrm{~kg}$ de cada aerogel/tonelada do licor negro;

- $4 \mathrm{~kg}$ de cada aerogel/tonelada do licor negro;

- $8 \mathrm{~kg}$ de cada aerogel/tonelada do licor negro.

O talco foi adicionado a um erlenmeyer de $50 \mathrm{~mL}$ contendo uma alíquota de $25 \mathrm{~mL}$ do licor com pitch e foi mantido por um período de agitação de 15 minutos a aproximadamente $100 \mathrm{rpm}$, utilizando-se, para tanto, um agitador magnético. Por sua vez, cada aerogel foi adicionado individualmente a um erlenmeyer com mesmo volume de licor, $25 \mathrm{~mL}$. Cada amostra foi agitada por um período de 15 minutos a aproximadamente $50 \mathrm{rpm}$, usando uma mesa agitadora a fim de evitar possíveis rupturas na estrutura do aerogel, ocasionadas por choques constantes com a barra magnética ("peixinho") no agitador.

Anteriormente às contagens das micelas das amostras contendo material adsorvente, talco e aerogel, foi contada, segundo a metodologia PAPTAC (2011), a quantidade de micelas coloidais do branco, ou seja, do licor negro sem adição de talco ou aerogel.

Após a agitação, utilizou-se uma pipeta automática para coletar alíquotas de $200 \mu \mathrm{L}$ das misturas, que foram alocadas nas câmaras de Neubauer, a fim de realizar a contagem das micelas dispersas de pitch. Cobriu-se a câmara com uma lamínula e, pela ação da capilaridade, o líquido movimentou-se para a região dos quadrados menores, onde foi feita a contagem das micelas. A Figura 2 mostra uma foto da câmara de Neubauer no momento de seu preenchimento com alíquota de amostra. 


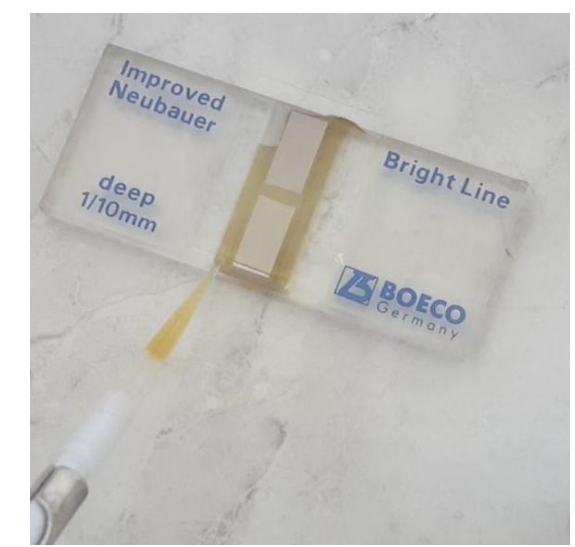

Figura 2 - Preenchimento da câmara de Neubauer.

Cada câmara de Neubauer possui dois compartimentos para leitura: um deles está representado pela Figura 3. Cada compartimento é dividido em nove quadrantes, cada qual com $1 \mathrm{~mm}^{2}$ de área. A contagem dos pitchs é feita no quadrante central. Este é subdividido em 25 quadrados, cada um com área de $0,04 \mathrm{~mm}^{2}$. Desses 25 , foram selecionados 5 quadrados para fazer a leitura, cada um subdividido em 16 quadrados menores, cada qual com área de $0,0025 \mathrm{~mm}^{2}$.

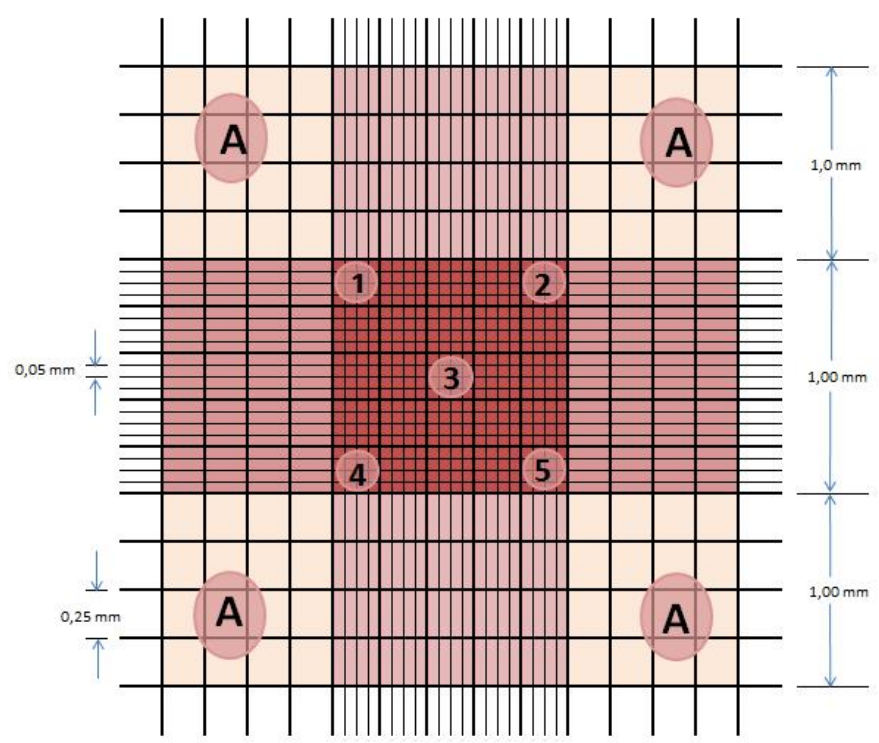

Figura 3 - Representação da câmara de Neubauer.

Com auxílio de um sistema de análise de imagens por vídeo microscopia computadorizada, conforme mostrado na Figura 4, e utilizando-se um microscópio com uma objetiva de 1000x de aumento, pôde ser feita a contagem das micelas dispersas, ou seja, as que não foram adsorvidas pelo talco ou pelos aerogéis nas amostras de licor. Um exemplo de pitch contabilizado está indicado na Figura 4. A contagem foi feita de maneira ágil de forma a evitar a evaporação do licor com consequente concentração de pitchs e perda de precisão do método. 


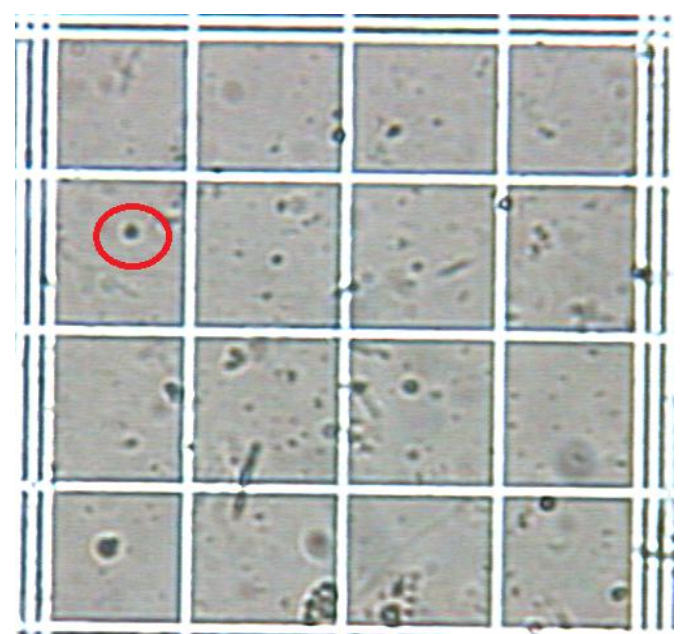

Figura 4 - Visão microscópica da área de contagem.

As contagens foram realizadas em três repetições para cada material. Algumas precauções e ações, citadas a seguir, foram aplicados ao longo das análises:

- Pela definição de pitch, consideramos na contagem somente esferas pequenas, de borda destacada e com movimento browniano;

- Foi mantido o foco do microscópio de forma que fossem identificados os pitchs dispersos no seio do licor, desconsiderando o fundo da câmara, onde se depositam outras sujeiras e a superfície.

A concentração de micelas ou partículas de pitch dispersas - CPD - foi determinada de acordo com a seguinte relação descrita na Equação 1:

$C P D=\frac{N \times K}{A \times H}$

Onde:

$\mathrm{CPD}=$ Concentração de partículas de pitch dispersas, $\left(\mathrm{N}^{\mathrm{o}} . / \mathrm{cm}^{3}\right)$;

$\mathrm{N}=$ Média do número de partículas dispersas, $\left(\mathrm{N}^{\circ}.\right)$;

$\mathrm{A}=$ Área do campo focal de análise na câmara de Neubauer, $\left(\mathrm{cm}^{2}\right)$;

$\mathrm{H}=$ Profundidade da câmara de Neubauer, $(\mathrm{cm})$;

$\mathrm{K}=$ Fator de correção de profundidade.

De acordo com a metodologia PAPTAC (2011), o fator $\mathrm{K}$ tem o valor de 1,1 para uma câmara de Neubauer com profundidade de $0,1 \mathrm{~mm}$. Esse fator é usado para corrigir a variação na profundidade da câmara e, consequentemente, no seu volume. Essa variação ocorre devido à tensão superficial promovida ao se cobrir a câmara com a lamínula no processo de contagem. 


\section{RESULTADOS E DISCUSSÃO}

Os resultados das análises das contagens de pitch das amostras de licor negro contendo talco como adsorvente e das amostras contendo os aerogéis como adsorventes são apresentados no Quadro 1. O desvio padrão foi calculado em relação às quantidades de pitch encontradas nas três repetições feitas para cada amostra. O CPD, concentração de partículas de pitch dispersas, foi calculado de acordo com a Equação 1.

Quadro 1 - Resultados das contagens de micelas de pitch coloidal dispersas - CPD $\left(\mathrm{N}^{\circ} . / \mathrm{cm}^{3}\right)$.

\begin{tabular}{|c|c|c|c|c|}
\hline Amostras & $\begin{array}{c}\text { Quantidade } \\
\text { (mg) }\end{array}$ & $\begin{array}{c}\text { Quantidade } \\
\text { média de } \\
\text { Pitch }\end{array}$ & $\begin{array}{c}\text { Desvio } \\
\text { Padrão }\end{array}$ & $\begin{array}{c}\text { CPD } \\
\text { (No. } / \mathbf{~ c m}^{3} \text { ) }\end{array}$ \\
\hline Branco & - & 49,20 & 0,75 & $2,16 \mathrm{E}+08$ \\
100:0 (CNF:Xilana) & 50 & 31,13 & 2,29 & $1,37 \mathrm{E}+08$ \\
100:0 (CNF:Xilana) & 100 & 23,27 & 3,34 & $\mathbf{1 , 0 2 E + 0 8}$ \\
100:0 (CNF:Xilana) & 200 & 26,67 & 0,38 & $1,17 \mathrm{E}+08$ \\
70:30 (CNF:Xilana) & 50 & 31,00 & 2,27 & $\mathbf{1 , 3 6 E + 0 8}$ \\
70:30 (CNF:Xilana) & 100 & 28,13 & 0,47 & $1,24 \mathrm{E}+08$ \\
70:30 (CNF:Xilana) & 200 & 26,33 & 2,93 & $1,16 \mathrm{E}+08$ \\
30:70 (CNF:Xilana) & 50 & 31,07 & 3,74 & $1,37 \mathrm{E}+08$ \\
30:70 (CNF:Xilana) & 100 & 29,47 & 0,34 & $1,30 \mathrm{E}+08$ \\
30:70 (CNF:Xilana) & 200 & 30,33 & 0,19 & $1,33 \mathrm{E}+08$ \\
\hline Branco & - & 50,73 & 3,50 & $2,23 \mathrm{E}+08$ \\
Talco & 51 & 20,73 & 1,09 & $9,12 \mathrm{E}+07$ \\
\hline
\end{tabular}

A Figura 5 apresenta a redução percentual na concentração das micelas de pitch de cada amostra, contendo seu respectivo material adsorvente, em relação ao branco e o Quadro 2 mostra os valores dos intervalos de confiança para cada valor médio de redução de pitchs, obtido a partir da utilização de cada agente adsorvente.

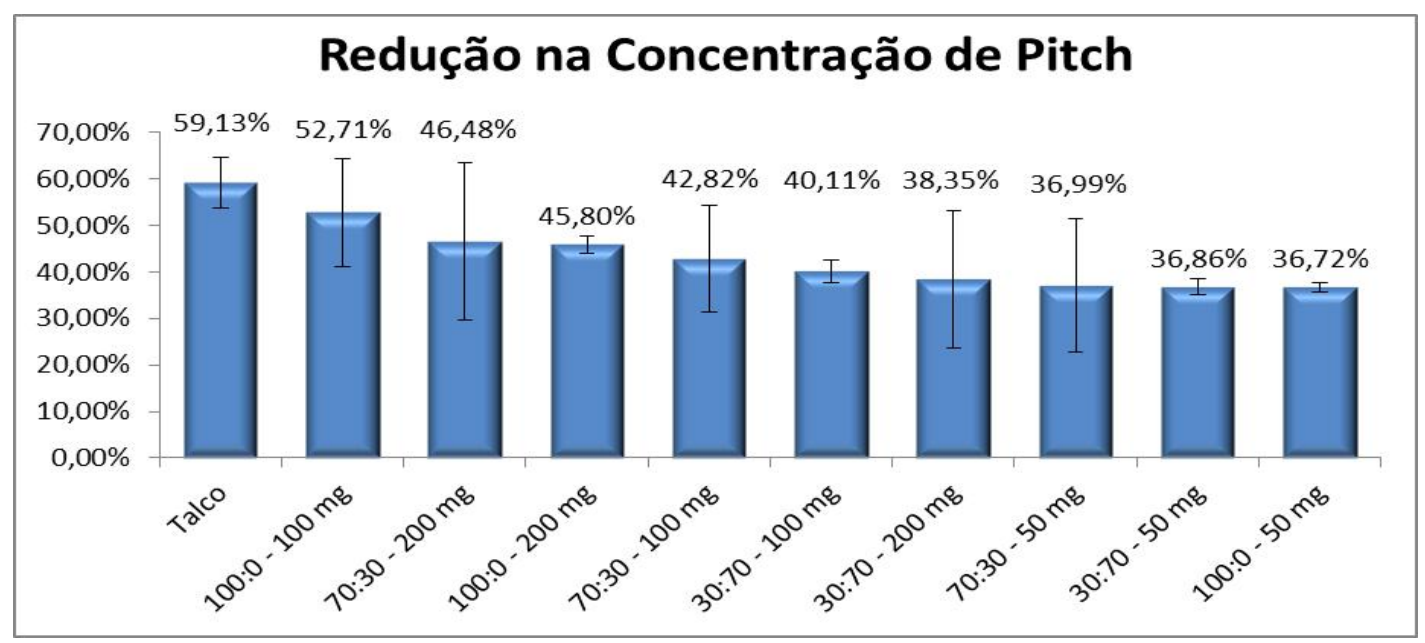

Figura 5 - Redução na concentração de pitch em cada amostra, \%, com respectivos intervalos de confiança.

Quadro 2 - Intervalos de confiança. 


\begin{tabular}{|c|c|}
\hline Amostra & $\begin{array}{c}\text { Intervalo de } \\
\text { confiança(\%) }\end{array}$ \\
\hline Talco & 5,33 \\
\hline $100: 0-50 \mathrm{mg}$ & 11,58 \\
$100: 0-100 \mathrm{mg}$ & 16,86 \\
$100: 0-200 \mathrm{mg}$ & 1,90 \\
$70: 30-50 \mathrm{mg}$ & 11,45 \\
$70: 30-100 \mathrm{mg}$ & 2,39 \\
$70: 30-200 \mathrm{mg}$ & 14,78 \\
\hline $30: 70-50 \mathrm{mg}$ & 14,30 \\
\hline $30: 70-100 \mathrm{mg}$ & 1,72 \\
\hline $30: 70-200 \mathrm{mg}$ & 0,96 \\
\hline
\end{tabular}

Observa-se pelo Quadro 2 que os intervalos de confiança (para $\alpha=0,05$ ) variaram bastante para cada amostra, assumindo altos valores em alguns casos. Isso se deve ao fato de o tamanho amostral ter sido pequeno (para cada amostra de adsorvente foram feitas três replicatas). Além disso, há erros empíricos inerentes ao experimento, que envolvem a contagem de pitchs, devido ao próprio processo de reconhecimento dos mesmos no microscópio e também pela variação de observadores durante as contagens. Sendo assim, assume-se que o intervalo de confiança aceitável nesse experimento para cada valor percentual médio de redução de pitchs é amplo, podendo ser considerado até $20 \%$.

Os materiais celulósicos são atrativos devido à grande capacidade de adsorção, origem renovável e biodegradabilidade. Particularmente, aerogéis feitos de materiais renováveis constituem uma interessante classe de adsorventes por apresentar uma combinação de baixa densidade, área superficial significativa e resiliência (GARCÍA-GONZÁLEZ et al., 2011). Isso explica o fato de que, independentemente de sua proporção CNF:Xilana, todos os aerogéis testados apresentaram poder de adsorção para o pitch.

A porosidade dos aerogéis, a existência de cargas e terminações negativas, devido ao grande número de hidroxilas presentes nas estruturas celulósicas e hemicelulósicas, e a presença de cargas positivas das resinas PAE contribuem para o grande poder de atração dos aerogéis sobre as micelas de pitch, que por sua vez, possuem caráter negativo (CÂMARA, 2003). Além disso, o movimento browniano do pitch também facilita sua adsorção. Ele permite a interação do pitch com diversos componentes do licor negro, que irão se incorporar à micela, facilitando o seu deslocamento à estrutura porosa e esponjosa dos aerogéis.

Uma primeira análise com relação à massa utilizada pode ser feita para cada tipo de aerogel separadamente. Para o aerogel com proporção 100:0 observa-se que o percentual de redução de uma amostra de $100 \mathrm{mg}$ foi a que mais se aproximou do poder de adsorção do talco, o qual é o produto referência usado na indústria usualmente. Nota-se que, um aumento da massa desse aerogel para $200 \mathrm{mg}$, não necessariamente levou a uma maior redução dos pitchs. Esse mesmo comportamento é observado no aerogel de composição 30:70. No entanto, o aerogel de composição 70:30 não apresentou esse comportamento. Neste caso, o aumento da massa do aerogel levou a um aumento do poder de adsorção. Como não se 
observa um comportamento padrão dos aerogéis, infere-se que deve existir, para cada tipo de aerogel, uma quantidade ótima de massa que adsorva o máximo de pitch, para uma mesma condição de experimento.

A análise através de microscopia eletrônica de varredura (MEV) e utilização do método Brunauer-Emmet-Teller (BET) foi feita por Silva (2015). Sob a ótica da estrutura microscópica reticular, quanto maior o teor de xilanas do aerogel, menor a sua porosidade, menor a profundidade dos poros dos aerogéis e, consequentemente, menor a sua área superficial comparativamente aos demais aerogéis utilizados no experimento, como demonstrado na Figura 6. Isso explica o fato de que, no geral, o aerogel com composição 30:70, independente da massa utilizada no experimento, foi o que menos apresentou poder de adsorção. Em contrapartida, os aerogéis com maior teor de CNF foram os que apresentaram um maior poder de adsorção, indo de encontro ao objetivo desse trabalho.

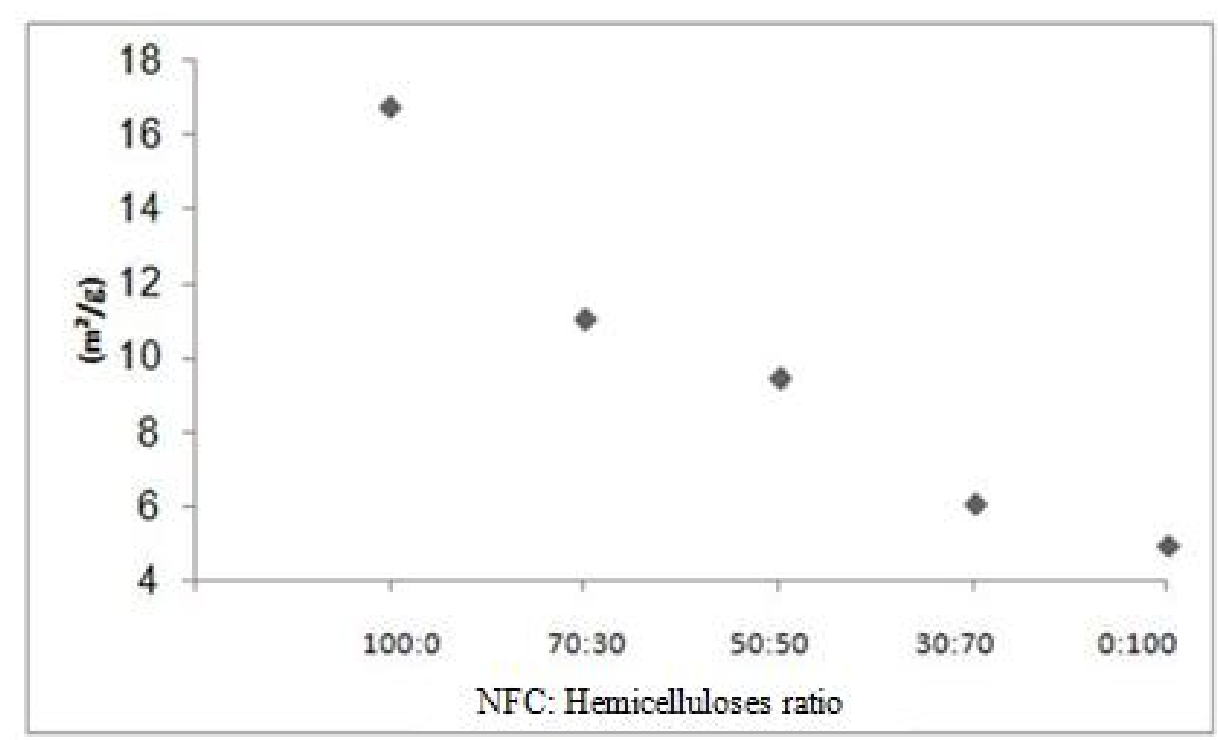

Figura 6 - Área superficial $\left(\mathrm{m}^{2} \cdot \mathrm{g}^{-1}\right)$ dos aerogéis em diferentes composições. Fonte: SILVA, 2015.

\section{CONCLUSÃO}

A partir dos experimentos realizados e análises dos resultados podem ser obtidas as seguintes conclusões:

Todos os aerogéis testados têm poder de adsorção de pitch, embora menores que o do talco, mineral usualmente utilizado para esse fim na indústria. Apesar disso, eles podem ser uma boa alternativa ao uso do talco, já que este é um material retirado diretamente da natureza, não renovável, enquanto os aerogéis são provenientes de processos de biorrefinaria, biodegradáveis e produzidos com celulose, polímero renovável e de maior abundância do planeta. Esses aerogéis também podem ser aplicados em diferentes etapas do processo da indústria celulósica, de modo que a adsorção total de pitchs seja bem efetiva.

Os aerogéis são altamente porosos, pouco densos e sua composição estrutural de celuloses, hemiceluloses e resina PAE, somada ao movimento browniano dos pitchs, 
estabelece uma condição favorável para a adsorção. Além disso, a partir de estudos mais aprofundados e aperfeiçoamento na estrutura dos aerogéis, com direcionamento da sua produção especificamente para a adsorção de pitch, pode haver otimização da capacidade de adsorção deles.

Os aerogéis com maior teor de CNF apresentaram um melhor poder de adsorção das micelas de pitch coloidal, devido a sua maior área superficial. Por sua vez, quanto maior o teor de xilanas, menor é a porosidade dos aerogéis, menor é a profundidade dos poros e assim, menor sua área superficial. Nesse contexto, o aerogel com massa de $100 \mathrm{mg}$ e proporção CNF:Xilana 100:0 foi o que apresentou o mais alto potencial de remoção de pitch, se aproximando do talco, produto referência na indústria. Enquanto isso, o aerogel com composição 30:70, apresentou, de modo geral, menor poder de adsorção de micelas de pitch, mesmo testando diferentes massas, em comparação aos aerogéis com maior teor de CNF.

Por fim, percebe-se por este estudo que os aerogéis não apresentaram comportamento que refletisse uma relação de proporcionalidade entre massa e potencial de adsorção, visto que um aumento na massa desses adsorventes não implicou, necessariamente, em um aumento na adsorção de micelas de pitch. Isso sugere a existência de uma quantidade ótima de aerogel, específica para cada tipo, que promova uma máxima adsorção.

Estudos futuros devem ser feitos para que se determine uma composição ideal de aerogel de polpa celulósica com propósito específico para a adsorção de pitch. Além disso, caberia também uma análise minuciosa a cerca dos pontos de atuação do aerogel dentro de uma indústria celulósica a fim de prever a melhor forma de diminuir o acúmulo dos pitchs nos equipamentos e nos produtos finais.

\section{REFERÊNCIAS}

AULIN, C.; NETRVAL J.; WAGBERG, L., et al. Aerogels from nanofibrillated cellulose with tunable oleophobicity. Soft Matter, v. 6, 3298-3305, 2010.

BARBOSA, L. C. A; CRUZ, M. P.; GOMIDE; J. L.; MALTHA, C. R. A; MILANEZ, A. F. Caracterização química do "pitch" em indústria de celulose e papel de Eucalyptus. Química Nova, v. 29, n. 3, 459-466, 2006.

CAMARA; A. L. Uso de talco nacional como aditivo adsorvente de piches e materiais pegajosos no processo de fabricação do papel. Dissertação (Mestrado). Universidade Federal de Minas Gerais, Belo Horizonte, 2003.

DEL RIO J. C.; GUTIERREZ, A.; GONZALEZ-VILLA, F. J.; MARTIN, F.; ROMERO, J. Characterization of organic deposits produced in the kraft pulping of Eucalyptus globulus wood. Journal of Chromatography A, v. 874, 457- 465, 1998.

DEL RIO J. C.; ROMERO, J.; GUTIERREZ, A. Analysis of pitch deposits produced in Kraft pulp mills using a totally free bleaching sequence. Journal of Chromatography A, v. 874, 235-245, 2000.

FORTUNATTO, A. C. Alternativas para o aproveitamento do licor negro da indústria de papel e celulose. Monografia. Universidade de São Paulo, São Paulo, 2014.

GARCÍA-GONZÁLEZ, C. A.; ALNAIEF, M.; Smirnova, I. Polysaccharide-based aerogels - Promising biodegradable carriers for drug delivery systems. Carbohydrate Polymers, v. 86, 1425-1438, 2011. 
GUTIERREZ, A.; DEL RIO, J. C.; MARTINEZ, M. J.; MARTINEZ, A. T. The biotechnological control of pitch in paper pulp manufacturing. Trends Biotechnology., v. 19, 340-348, 2001.

HABIBI, Y.; LUCIA, L. A.; ROJAS, O. J. Cellulose nanocrystals: chemistry, selfassembly, and applications. Chemical Reviews, v. 110, n. 6, 3479-3500, 2010.

JORDÃO, M.C.S. Pitch na Indústria de Celulose e Papel. Instituto de Pesquisas Tecnológicas - IPT, São Paulo, 1991.

O PAPEL. ISSN 0031-1057. Ano 77, n. 5, 18-19, São Paulo, maio 2016.

PÄÄKKÖ et al. Enzymatic Hydrolysis Combined with Mechanical Shearing and HighPressure Homogenization for Nanoscale Cellulose Fibrils and Strong Gels. Biomacromolecules, v. 8, n. 6, 1934-1941, 2007.

Pulp And Paper Technical Association of Canada (PAPTAC) usefull method G.12U. Determination of dispersed Pitch. 2011.

SANSÍGOLO, C. A.; SARTO, C. Cinética da remoção dos extrativos da madeira de Eucalyptus grandis durante polpação Kraft. Acta Scientiarum, Agronomy, v. 32, n. 3, 227-235, Maringá, 2010.

SILVA, J. C. Biorefinery of lignocellulosic materials: novel products, methods and applications of forest and agricultural feedstocks. 83 f. Tese (Doutorado). Universidade Federal de Viçosa, Minas Gerais, 2015.

\title{
AEROGELS APPLICATION TO REMOVE PITCH IN THE PULP AND PAPER INDUSTRY
}

\begin{abstract}
Black liquor formed in the pulping of pulp and paper industry contains pitchs, colloidal resinous materials and wood derivatives that accumulate in machinery of the pulp industry, damaging equipment or the final product. The objective of this work was to study the use of cellulose-based aerogels in pitch removal, a function of talc in most industries. Aerogels are porous solids, produced from lyophilization of a colloidal suspension of nanofibrillated cellulose and xylans, in different proportions, and PAE resin. The pitch counts were made using a Neubauer camera. All the aerogels presented adsorption power, although higher the CNF content, better the adsorbent. In general, 100:0 aerogels (CNF: Xylan) are better adsorbents compared to 30:70 aerogels. No proportionality was observed between mass and adsorption capacity, suggesting the existence of specifics amounts for each aerogel.
\end{abstract}

KEYWORDS: Pitch; Aerogel; Cellulose; Hemicellulose; Adsorption. 